\title{
Dissociation between the Effects of Theophylline and Caffeine on Premature Airway Smooth Muscle
}

\author{
PRABHU N. MEHTA,' HOWARD B. PANITCH, MARLA R. WOLFSON, AND \\ THOMAS H. SHAFFER \\ Department of Physiology, Temple University School of Medicine and Department of Pediatrics, St. Christopher's \\ Hospital for Children, Philadelphia, Pennsylvania 19134-1095
}

\begin{abstract}
Xanthine derivatives relax adult airway smooth muscle (ASM). To determine whether caffeine and theophylline relax preterm ASM contracted by acetylcholine, 27 tracheal rings obtained from seven preterm lambs (120-135 d gestation) were studied. ASM was contracted using $10^{-5} \mathrm{M}$ acetylcholine (control) after the muscle was stretched to the length at which maximum active tension was developed isometrically. Concentration-effect curves for each xanthine were obtained by cumulative addition of the drug. Theophylline produced a significant decrease ( $p$ $<0.001$ ) in active tension at each dose, whereas caffeine significantly increased $(p<0.001)$ active tension at $10^{-4}$ and $10^{-3} \mathrm{M}$ concentrations. Addition of caffeine and theophylline to previously uncontracted ASM did not alter tension. Thus, it appears that, in contrast to their effect on adult ASM, the xanthine derivatives caffeine and theophylline have differential effects on prestimulated ASM in preterm lambs. These findings raise important questions about various aspects of the current therapeutic use of caffeine and theophylline. (Pediatr Res 29: 446-448, 1991)
\end{abstract}

\section{Abbreviations}

ASM, airway smooth muscle

Ach, acetylcholine

KBS, Kreb's buffer solution

$\mathrm{SR}$, sarcoplasmic reticulum

Xanthine derivatives, theophylline and caffeine, are commonly used in the management of apnea of prematurity (1) They are known to stimulate CNS respiratory centers (1) and skeletal muscle (2), but they are also proposed to relax ASM (3)

Differences in sensitivity of ASM to various agonists as a function of postnatal age have been reported in several animal studies (4-6). Theophylline and caffeine are known to relax adult ASM (3) and have been shown to decrease bronchomotor tone in infants suffering from bronchopulmonary dysplasia $(7,8)$. However, very little is known about the direct effects of theophylline and caffeine on preterm ASM.

Infants who receive theophylline and caffeine drugs for apnea of prematurity may also have coexisting increased bronchomotor

Received March 22, 1990; accepted December 6, 1990.

Correspondence: Prabhu N. Mehta, M.D., c/o Thomas H. Shaffer, Ph.D. Temple University School of Medicine, Dept. of Physiology and Pediatrics, 3420

N. Broad St., Philadelphia, PA 19140.

Supported in part by Grant BRSG S07 RR05417 from the Division of Research Resources of NIH and NIH HL 32301

${ }^{1}$ Currently on staff in the Division of Neonatology, I-3, Henry Ford Hospital 2799 W. Grand Blvd., Detroit, MI 48202. tone. As previously shown, alterations in ASM tone can profoundly affect the pattern of air flow, dead space volume, and compliance of airway and lung tissue $(4,9,10)$. In this regard, it would be important to know the direct effects of these commonly used antiapneic drugs on the bronchomotor tone of preterm ASM

The purpose of our study was to evaluate and compare the effect of theophylline and caffeine on preterm ASM tone. As a model of ASM during early development, isolated segments of tracheal smooth muscle from preterm lambs were studied in vitro to determine the direct action of these agents independent of extrinsic neural or humoral effects.

\section{MATERIALS AND METHODS}

Adult pregnant ewes, sedated with ketamine $\mathrm{HCl}, 500 \mathrm{mg}$ intramuscularly, were given epidural anesthesia with $0.5-1.0 \mathrm{mg} /$ $\mathrm{kg}$ of $0.75 \%$ Bupivicaine (Marcaine; ASTRA Pharmaceuticals, Westborough, MA). Preterm lambs between 120 and $135 \mathrm{~d}$ postconceptional age were subsequently delivered by uterotomy as previously described (11).

Cervical tracheal segments were removed after euthanization and stored in KBS (composition in $\mathrm{mM}: \mathrm{NaCl} 120, \mathrm{KCl} 5, \mathrm{CaCl}_{2}$ 2.5, $\mathrm{MgCl}_{2}$ 1.2, glucose 11.5, $\left.\mathrm{NaHCO}_{3} 22, \mathrm{NaH}_{2} \mathrm{PO}_{4} 1.2\right), \mathrm{pH}$ $7.40 \pm 0.05$, at $13^{\circ} \mathrm{C}$. Two- to $3-\mathrm{mm}$ wide tracheal rings were cut from the cervical tracheal segments. The rings were opened and the study performed after placing these rings in individual muscle baths filled with $\mathrm{KBS}\left(37^{\circ} \mathrm{C}\right)$, through which a mixture of $95 \%$ $\mathrm{O}_{2}$ and $5 \% \mathrm{CO}_{2}$ was continuously bubbling. One cartilaginous end of the ring was attached to the metal rod and the other to a force transducer (Grass FTO3C; Grass Instruments, Quincy, $\mathrm{MA}$ ) by means of a wire whose compliance was negligible over the range of forces measured. The wire was tied to the ring via 3-0 surgical silk. In this way, the wire could be easily attached at one insertion and the ring fixed at the other insertion with no stray compliance in the system. The force transducer was attached to a screw micrometer calibrated in $0.5-\mathrm{mm}$ increments, which, when moved, caused an equal lengthening of the muscle strip that bridged the cartilaginous ends of the tracheal ring. Rings were equilibrated under zero load for $30 \mathrm{~min}$ before the study.

Twenty-seven tracheal rings from seven preterm lambs were studied. Tension was recorded on a Grass (7PI) multichannel recorder. The rings were stretched until resting or passive tension was just noted. Once passive tension had reached stable level, $10^{-5} \mathrm{M}$ Ach was added to the bath. The muscle was allowed to contract isometrically, and total tension was measured once it reached a plateau. Active tension was calculated as the difference between total and passive tension. The ring was then stretched in a stepwise fashion, in $0.5-\mathrm{mm}$ increments. After each step, passive, total, and active tension were measured as above. The 
length at which maximal active tension developed $\left(\mathrm{L}_{0}\right)$ was determined for each ring. Twenty-one rings, set at their predetermined $\mathrm{L}_{0}$, were studied after stimulation with a submaximal dose of Ach $\left(10^{-5}\right)$. When active tension reached a plateau (control tension), concentration-effect curves were obtained by cumulative $\left(10^{-7}\right.$ to $\left.10^{-5} \mathrm{M}\right)$ addition of theophylline or caffeine (randomized order) to the muscle bath. After obtaining concentration-effect curves for each drug, the rings were repeatedly washed with fresh KBS until they returned to their prestimulated resting tension. The effects of both theophylline and caffeine were studied on each ring. Concentration-effect curves for both caffeine and theophylline were also elicited from six rings from two preterm airways not previously stimulated by Ach.

Tension at each drug dose was expressed as a percent of control tension. A two-way, repeated measures analysis of variance was used to evaluate tension as a function of dose and drug; when significance was determined ( $p<0.05$ level), Tukey and paired $t$ tests were performed post hoc to identify significant differences between means. In addition, Bartlett's test was performed to test for differences in variances among the groups.

\section{RESULTS}

Stimulation with a submaximal dose of Ach $\left(10^{-5}\right)$ yielded a tension of $60-75 \%$ maximum tension for all rings and remained stable for a period of $45 \mathrm{~min}$. As shown in Table 1, theophylline produced a significant $(p<0.01)$ dose-related decrease in active tension of these rings. In addition, active tension was significantly $(p<0.001)$ less than baseline at each dose of theophylline. As noted by the error terms, there was little variation in active tension across rings and animals, and all animals demonstrated a consistent trend of decreasing tension with increasing theophylline dose.

In contrast, as shown in Table 2, caffeine yielded a significant $(p<0.05)$ dose-related increase in active tension of the Ach preconstricted rings, with all animals demonstrating a consistent response. Moreover, there was a further significant $(p<0.001)$ increase in active tension after stimulation with $10^{-4}$ and $10^{-5}$ $\mathrm{M}$ caffeine. As with theophylline, there was little variation in active tension across rings and animals.

These results demonstrate a significant difference in active tension between drugs over the range of $10^{-6}$ to $10^{-3} \mathrm{M}$. This difference in concentration effect is best illustrated in Figure 1. As shown, as the concentration of caffeine was increased, there was a gradual potentiation of active tension, which sharply increased at $10^{-5} \mathrm{M}$. The concentration effect of theophylline mirrored the caffeine response in that there was a gradual attenuation followed by a sharp decrease in active tension at $10^{-5} \mathrm{M}$.

There were no apparent concentration effects of either theophylline or caffeine or ASM of unstimulated tracheal rings.

\section{DISCUSSION}

Xanthines are known to alter function of the cardiovascular, neuroendocrine, and immune systems (3). To avoid these systemic effects, studies were performed in vitro using isolated tracheal rings so that the direct effects of these drugs on ASM tone could be examined (6). As a rule, every drug has the potential for species variations in addition to quantitative and qualitative differences within airway subdivisions. However, there is ample precedent for using tracheal muscle as a model for developing ASM $(4,6,10,12)$.

Theophylline has the ability to relax adult ASM if it is contracted experimentally by Ach and histamine or spontaneously, as in asthma $(3,13)$. Studies of infants suffering from bronchopulmonary dysplasia showed that theophylline produces a bronchodilator response (8). In our experiment, theophylline decreased active tension of Ach-stimulated ASM at subtherapeutic $\left(10^{-7}\right.$ to $\left.10^{-5} \mathrm{M}\right)$, therapeutic $\left(10^{-4} \mathrm{M}\right)$, and toxic $\left(10^{-3} \mathrm{M}\right)$ concentrations. Approximately $30 \%$ reduction in active tension of stimulated ASM was seen at $10^{-4} \mathrm{M}$ concentration of theophylline. Theophylline was capable of decreasing tension at all doses, reconfirming its bronchodilator effect on preterm ASM.

In vitro studies of adult ASM preparations (13) as well as studies in adult and pediatric asthmatic patients $(14,15)$ have shown caffeine to have a bronchodilator effect. Currently, data on the direct effects of caffeine on preterm ASM are not available. In our experiment, caffeine increased active tension of Achstimulated ASM at therapeutic $\left(10^{-4} \mathrm{M}\right)$ and toxic $\left(10^{-3} \mathrm{M}\right)$ concentrations. Active tension increased by approximately 15 and $65 \%$ at the $10^{-4}$ and $10^{-3} \mathrm{M}$ concentrations of caffeine, respectively.

It has been suggested that release of calcium from SR to the myofibrils initiates actin-myosin cross-linkage, and reuptake of calcium by the free SR causes relaxation (16). Caffeine has been shown to increase vascular and cardiac smooth muscle as well as skeletal muscle tone by inducing release of calcium from intracellular storage sites (16-18). Studies have also shown that caffeine enhances the sensitivity of the calcium-induced calcium release mechanism at the cellular level in vascular, cardiac, and skeletal muscles $(16-18)$.

Theophylline and caffeine produced opposing responses, suggesting a difference in their mechanism of action on preterm ASM. In our experiment, caffeine-induced tension increase was seen only in Ach-stimulated muscles, raising the possibility that postcaffeine ASM tension increase could have been due to the enhancement of the Ach-induced calcium release from the SR. A study done by Kobayashi et al. (19) on rat arterial smooth muscle showed that caffeine may have a depolarization-sensitive intracellular calcium storage site identical to one already known for potassium. Biochemically, compounds of xanthine class inhibit phosphodiesterase activity with a resultant increase in

Table 1. Effect of theophylline on airway smooth muscle tension (\% control)*

\begin{tabular}{lccccc}
\hline & \multicolumn{5}{c}{ Dose (Log M) } \\
\cline { 2 - 5 } Animal & -7 & -6 & -5 & -4 & -3 \\
\hline $1(4)$ & 90.5 & 88.0 & 85.6 & 61.9 & 32.1 \\
$2(2)$ & 100.0 & 95.6 & 91.5 & 74.5 & 47.4 \\
$3(3)$ & 95.0 & 90.2 & 81.9 & 64.3 & 27.8 \\
$4(2)$ & 93.7 & 88.3 & 83.2 & 64.0 & 39.0 \\
$5(2)$ & 93.4 & 86.8 & 84.0 & 64.1 & 36.7 \\
$6(4)$ & 95.4 & 91.4 & 90.5 & 78.4 & 50.2 \\
$7(4)$ & 96.9 & 94.5 & 87.0 & 67.3 & 50.1 \\
Mean (7) $\dagger$ & $95.0 \pm 1.1$ & $90.7 \pm 1.3$ & $86.2 \pm 1.4$ & $69.2 \pm 2.7$ & $40.5 \pm 3.4$ \\
Mean $(21) \ddagger$ & $94.8 \pm 1.2$ & $90.8 \pm 1.3 \S$ & $86.4 \pm 1.5 \S$ & $69.9 \pm 2.4 \S$ & $40.8 \pm 2.6 \S$ \\
\hline
\end{tabular}

\footnotetext{
* Control tension based on $10^{-5} \mathrm{M}$ Ach stimulation; number in parentheses indicates the number of rings or animals.

$\dagger$ Mean \pm SEM values for all animals.

$\ddagger$ Mean \pm SEM values for all rings.

$\S$ Significant difference from control tension $(p<0.001)$ and significant difference from caffeine value $(p<0.001)$
} 
Table 2. Effect of caffeine on airway smooth muscle tension (\% control)*

\begin{tabular}{|c|c|c|c|c|c|}
\hline \multirow[b]{2}{*}{ Animal } & \multicolumn{5}{|c|}{ Dose $(\log M)$} \\
\hline & -7 & -6 & -5 & -4 & -3 \\
\hline $1(4)$ & 97 & 101.1 & 103.6 & 114.6 & 148.8 \\
\hline $2(2)$ & 102.5 & 105.5 & 108.8 & 106.7 & 142.8 \\
\hline $3(3)$ & 100 & 100 & 100 & 110.7 & 175.3 \\
\hline $4(2)$ & 98.5 & 98.6 & 100 & 136.5 & 190.6 \\
\hline $5(2)$ & 98.2 & 100 & 100 & 117.3 & 194.7 \\
\hline $6(4)$ & 95.6 & 94.5 & 94.9 & 109.6 & 173.8 \\
\hline $7(4)$ & 101.2 & 101.2 & 101.9 & 112.4 & 151 \\
\hline Mean (7) $\dagger$ & $99.0 \pm 0.9$ & $100.1 \pm 1.2$ & $101.3 \pm 1.6$ & $115.4 \pm 3.7$ & $168.1 \pm 7.9$ \\
\hline Mean $(21) \ddagger$ & $98.8 \pm 0.9$ & $99.9 \pm 1.2 \S$ & $100.9 \pm 1.3 \S$ & $114.3 \pm 2.1 \S \|$ & $165.5 \pm 4.9 \S \|$ \\
\hline
\end{tabular}

* Control tension based on $10^{-5} \mathrm{M}$ Ach stimulation; number in parentheses indicates the number of rings or animals.

$\dagger$ Mean \pm SEM values for all animals.

$\$$ Mean \pm SEM values for all rings.

$\S$ Significant difference from theophylline value $(p<0.001)$.

\| Significant difference from control tension $(p<0.001)$.

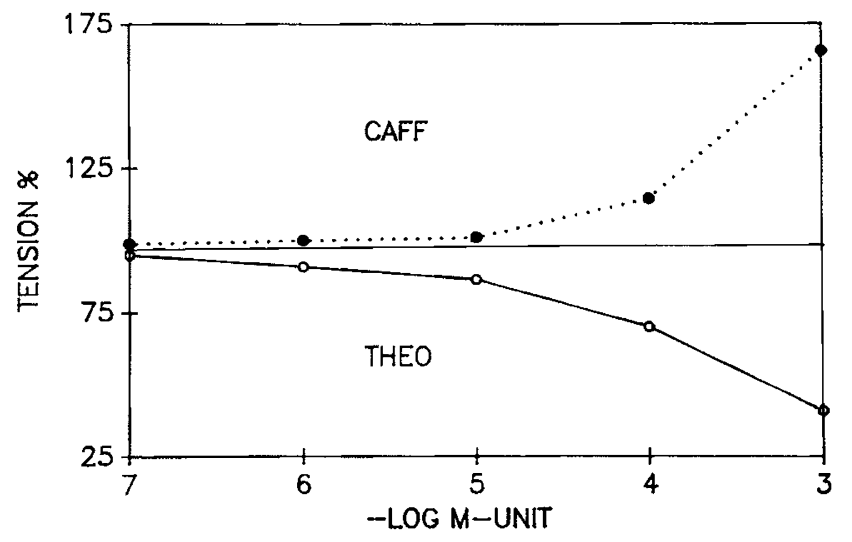

Fig. 1. Concentration-effect curves for theophylline (THEO) and caffeine $(C A F F)$.

cAMP concentration, mobilize intracellular calcium, and competitively inhibit adenosine receptors (20). The degree to which theophylline demonstrates these effects at therapeutic concentrations is limited to inhibition of adenosine receptors. There is no simple relationship between these effects and the type of xanthine. Recent studies have shown that the relative contribution of various effects of xanthine derivatives differs from compound to compound $(13,21)$. Therefore, it is possible that caffeine and theophylline have relative dominance of one particular effect over another and thereby show a differential effect on ASM tone. Further experiments are needed to determine the mechanism of caffeine-induced ASM tension increase observed in our study.

Finally, ASM contraction has been shown to decrease compliance of the neonatal airway $(4,10)$. By decreasing the airway compliance, contraction of ASM may also decrease airway resistance under conditions of high transmural pressure (10). It may be possible that theophylline increases deformation of the compliant preterm airway by decreasing ASM tone, whereas caffeine may stabilize preterm airways by increasing the tone of ASM. Further work is in progress in this laboratory to evaluate the effects of xanthines on ASM in in vitro and in vivo models (12).

In conclusion, our study demonstrates that theophylline decreases the tone of stimulated preterm ASM, whereas caffeine increases their tone. Neither xanthine altered the tone of nonstimulated preterm ASM. These results raise an important question about various aspects of the current therapeutic use of caffeine and theophylline in neonates with increased bronchomotor tone.

\section{REFERENCES}

1. Aranda JV, Turmen T 1979 Methylxanthines in apnea of prematurity. Clin Perinatol 6:87-108

2. Supinski AS, Chandler D, Kelson SG 1984 The effects of caffeine and theophylline on diaphragm contractility. Am Rev Respir Dis 130:429-433

3. Rall TW 1985 Central nervous system stimulants: the methylxanthines. In: Gillman AG, Goodman LS, Rall TW, Murad F (eds) The Pharmacological Basis of Therapeutics. MacMillan Publishing Co, New York, pp 589-603

4. Koslo RJ, Bhutani VK, Shaffer TH 1986 The role of tracheal smooth muscle contraction on neonatal tracheal mechanics. Pediatr Res 20:1216-1220

5. Hayashi S, Toda N 1980 Age related alterations in the response of rabbit tracheal smooth muscle to agents. J Pharmacol Exp Ther 214:675-681

6. Panitch HB, Allen JL, Ryan JP, Wolfson MR, Shaffer TH 1989 A comparison of preterm and adult airway smooth muscle mechanics. J Appl Physiol 66:1769-1765

7. Monin P, Vert P 1987 The management of bronchopulmonary dysplasia. Clin Perinatol 14:531-549

8. Rooklin AR, Moomjian AS, Shutack JG, Schwartz JG, Fox WW 1979 Theophylline therapy in bronchopulmonary dysplasia. J Pediatr 95:882-885

9. Wolfson MR, Shaffer TH 1990 The differential effects of pancuronium bromide on cardiopulmonary function in the neonatal lamb. Pediatr Pulmonol 8:233-239

10. Penn RB, Wolfson MR, Shaffer TH 1988 Effect of tracheal smooth muscle tone on the collapsibility of extremely immature airways. J Appl Physiol 65:863-869

11. Shaffer TH, Delivoria-Papadopoulos M, Arcinue E, Paez P, Dubois AB 1976 Pulmonary mechanics in premature lambs during the first few hours of life. Respir Physiol 28:179-188

12. Keklikian E, Wolfson MR, Roache $\mathrm{R}$, Shaffer TH 1990 In vivo effects of caffeine on airway reactivity in the neonatal lamb. Pediatr Res 27:206A(abstr)

13. Jeppsson AB, Waldeck JB, Johansson U 1982 Dissociation between the effects of some xanthine derivatives on the tracheal smooth muscle and on the skeletal muscle. Acta Pharmacol Toxicol 51:115-121

14. Bukowsky JM, Hakatsu K 1987 The bronchodilator effect of caffeine in adult asthmatics. Am Rev Respir Dis 135:173-175

15. Becker AB, Simons KJ, Gillespie CA, Simons FER 1984 The bronchodilator effects and pharmacokinetics of caffeine in asthma. N Engl J Med 310:743746

16. Kuriyama H, Ito Y, Suzuki H, Kitamura K, Itoh T 1982 Factors modifying contraction relaxation cycle in vascular smooth muscles. Am $\mathbf{J}$ Physiol 243:H64I-H662

17. Martonosi A 1984 Mechanisms of calcium release from sarcoplasmic reticulum of skeletal muscle. Physiol Rev 64:1240-1306

18. Fabiato A, Fabiato F 1977 Calcium release from sarcoplasmic reticulum. Circ Res 40:119-129

19. Kobayashi S, Kanaide H, Nakamara M 1986 Complete overlap of caffeine and $\mathrm{K}^{+}$depolarization sensitive intracellular calcium storage site in culture rate arterial smooth muscle cells. J Biol Chem 261(33):15709-15713

20. Bukowskyj M, Natkatsu K, Munt PW 1984 Theophylline reassessed. Ann Intern Med 101:63-73

21. Kramer AL, Wells JN 1980 Xanthines and skeletal muscle: lack of relationship between phosphodiesterase inhibition and increased twitch tension in rat diaphragm. Mol Pharmacol 17:73-78 